\title{
Effects of renalase deficiency on liver fibrosis markers in a nonalcoholic steatohepatitis mouse model
}

\author{
KATSUYUKI TOKINOYA ${ }^{1}$, NANAMI SEKINE ${ }^{2}$, KAI AOKI ${ }^{1}$, SEIKO ONO ${ }^{2}$, TOMOAKI KUJI ${ }^{1}$, \\ TAKEHITO SUGASAWA ${ }^{3}$, YASUKO YOSHIDA ${ }^{4}$ and KAZUHIRO TAKEKOSHI ${ }^{3}$
}

\begin{abstract}
${ }^{1}$ Doctoral Program in Sports Medicine, Graduate School of Comprehensive Human Sciences, University of Tsukuba, Tsukuba, Ibaraki 305-8577; ${ }^{2}$ Graduate School of Comprehensive Human Sciences, University of Tsukuba, Tsukuba, Ibaraki 305-8574;

${ }^{3}$ Division of Clinical Medicine, Faculty of Medicine, University of Tsukuba, Tsukuba, Ibaraki 305-8577; ${ }^{4}$ Department of

Clinical Laboratory Science, Faculty of Health Sciences, Tsukuba International University, Tsuchiura, Ibaraki 300-0051, Japan
\end{abstract}

Received August 24, 2020; Accepted October 6, 2020

DOI: $10.3892 / \mathrm{mmr} .2021 .11849$

\begin{abstract}
Progression of nonalcoholic steatohepatitis (NASH) is attributed to several factors, including inflammation and oxidative stress. In recent years, renalase has been reported to suppress oxidative stress, apoptosis and inflammation. A number of studies have suggested that renalase may be associated with protecting the liver from injury. The present study aimed to clarify the effects of renalase knockout (KO) in mice with NASH that were induced with a choline-deficient high-fat diet (CDAHFD) supplemented with $0.1 \%$ methionine. Wild type (WT) and KO mice (6-week-old) were fed a normal diet (ND) or CDAHFD for 6 weeks, followed by analysis of the blood liver function markers and liver tissues. CDAHFD intake was revealed to increase blood hepatic function markers, lipid accumulation and oxidative stress compared with ND, but no significant differences were observed between the WT and KO mice. However, in the KO-CDAHFD group, the Adgrel and $T g f b 1$ mRNA levels were significantly higher, and $\alpha$-SMA expression was significantly lower compared with the WT-CDAHFD group. Furthermore, the Gclc mRNA and phosphorylated protein kinase B (Akt) levels were significantly lower in the KO-ND group compared with the WT-ND group. The results of the current study indicated that as NASH progressed in the absence of renalase, oxidative stress, macrophage infiltration and TGF- $\beta$ expression were enhanced, while
\end{abstract}

Correspondence to: Professor Kazuhiro Takekoshi, Division of Clinical Medicine, Faculty of Medicine, University of Tsukuba, 1-1-1 Tennodai, Tsukuba, Ibaraki 305-8577, Japan

E-mail:k-takemd@md.tsukuba.ac.jp

Abbreviations: KO, knockout; WT, wild type; NASH, nonalcoholic steatohepatitis; CDAHFD, choline-deficient high-fat diet; ND, normal diet

Key words: transforming growth factor- $\beta, \alpha$-smooth muscle actin, Akt, oxidative stress, liver, renalase
$\alpha$-SMA expression in NASH may be partly suppressed due to the decreased phosphorylation of Akt level.

\section{Introduction}

Nonalcoholic steatohepatitis (NASH) is a progressive liver disease characterized by steatosis, inflammation, and fibrosis leading to liver cirrhosis and cancer (1). NASH risk factors include obesity, hypertension, and abnormal lipid metabolism (2). Hepatic steatosis results from the accumulation of some lipids. Free fatty acid delivery to the liver accounts for almost two-thirds of the lipid accumulation (3). The two-hit hypothesis, which states that hepatitis is induced by inflammation and oxidative stress after lipid accumulation in hepatocytes, has been considered the main mechanism underlying NASH progression (4). Recently, the multiple parallel hit hypothesis, which states that hepatitis progresses due to several factors that lead to fat accumulation in the liver, adipose tissue, and digestive tract, has gained support (3). The activation of hepatic stellate cells by increased production of transforming growth factor beta (TGF- $\beta$ ) by Kupffer cells and hepatocytes; activation of inflammatory cytokines such as the tumor necrosis factor- $\alpha$ (TNF- $\alpha$ ), interleukin (IL)-6, and IL-1 $\beta$ due to oxidative stress; and acceleration of hepatocyte apoptosis contribute to the progression of NASH. In addition, these inflammatory cytokines are regulated by nuclear factor- $\kappa \mathrm{B}$ $(\mathrm{NF}-\kappa \mathrm{B})$. Furthermore, the progression of liver fibrosis in $\mathrm{NASH}$ is associated with alterations in the extracellular matrix mainly by increased production of type I collagen (5-9). NASH progresses with increasing degrees of fibrosis, with the most common complication being cirrhosis (2).

Renalase is a flavin adenine dinucleotide-dependent amine oxidase that metabolizes catecholamines such as dopamine, epinephrine, and norepinephrine (10). Renalase is secreted by the proximal tubule in the kidney into the blood to degrade catecholamines and regulate blood pressure $(10,11)$. One study has reported that patients with chronic kidney disease and type II diabetes have high renalase levels in the blood (12). Renalase activates the PI3K/Akt and extracellular signal-regulated kinase signalling pathways and exerts cell-protective effects via the renalase receptor plasma membrane $\mathrm{Ca}^{2+}$ 
ATPase isoform $4 \mathrm{~b}$ (PMCA4b) $(13,14)$. Wu et al showed that TGF- $\beta$ increases the levels of fibrosis markers such as $\alpha$-smooth muscle actin ( $\alpha$-SMA), E-cadherin, and collagen type I $\alpha 1$ (Colla1) in human kidney 2 cells (15). In addition, hepatic renalase expression increases in response to oxidative stress in the mouse liver with induced ischemia-reperfusion (IR) injury, suggesting that renalase protects tissues (16). Recently, the downregulation of renalase in nonalcoholic fatty liver disease (NAFLD) and its protective role against liver IR injury has been reported, indicating that the downregulation of renalase may be involved in the susceptibility of the fatty liver to IR injury (17).

The progression of liver diseases such as NAFLD and NASH is considered to be suppressed by the inhibitory effects of renalase on inflammation, oxidative stress, and apoptosis. Therefore, we hypothesized that if NASH is induced in the absence of renalase, we should be able to observe increased liver inflammation and fibrosis and a significant decline in the liver function. In order to clarify this, we fed renalase knockout (KO) mice with a choline-deficient high-fat diet (CDAHFD) supplemented with $0.1 \%$ methionine to induce NASH. We then characterized the effects of the renalase $\mathrm{KO}$ on the mouse liver using several markers of liver health and activity.

\section{Materials and methods}

Animals and experimental design. B6;129S1-Rnlstm1Gvd/J mice were purchased from the Jackson Laboratory. Renalase KO and wild type (WT) mice were produced by mating heterozygous mice and identified by polymerase chain reaction (PCR) amplification of mouse genomic DNA, as previous described (7). A total of 24 male mice $(\mathrm{KO}, \mathrm{n}=12$; WT, $n=12$ ) were fed standard chow and water ad libitum and housed under standard laboratory conditions $\left(23.5 \pm 2.5^{\circ} \mathrm{C}\right.$, $52.5 \pm 12.5 \%, 14: 10$-h light-dark cycle). At 6 weeks of age, these mice were divided into four groups; WT-normal diet (ND; MF $12 \mathrm{~mm} \phi$ pellet; Oriental Yeast Co.), WT-choline-deficient, L-amino acid-defined, high-fat diet (CDAHFD; A06071302, Research Diets), KO-ND, and KO-CDAHFD. The diet was replenished every 3-4 days. After 6 weeks under each condition, the mice were killed by cervical dislocation after anesthesia with $5 \mathrm{ml}$ of the mix solution (isoflurane:propylene glycol=3:7) added to the container of approximately $3 \mathrm{~L}$. The current study followed the ethical guidelines from western institutions to justify the method of euthanasia used in the study. In addition, the volume of propylene glycol used in the current study exhibited no toxicity (18). Liver samples and blood from the inferior vena cava were collected. The sera were collected by centrifugation $\left(4^{\circ} \mathrm{C}, 3,000 \mathrm{x} \mathrm{g}, 30 \mathrm{~min}\right)$ after settling for $16 \mathrm{~h}$ at $4^{\circ} \mathrm{C}$. Samples were stored at $-80^{\circ} \mathrm{C}$ for subsequent analyses.

Assay for liver cholesterol and triglyceride and serum hepatic markers in the blood. Total cholesterol (T-CHO) and triglyceride (TG) in the liver were analysed by Cosmo Bio Co., Ltd. Serum aspartate aminotransferase (AST), alanine aminotransferase (ALT), alkaline phosphatase (ALP), and lactate dehydrogenase (LDH) were analysed by Fujifilm Bio Inc., Ltd.

Immunostaining. Liver samples were replaced with $70 \% \mathrm{EtOH}$ overnight after fixation in $10 \%$ paraformaldehyde in PBS for $72 \mathrm{~h}$. The liver tissue was cut into $3-\mu \mathrm{m}$ thick sections. Hematoxylin and eosin (H\&E) and Masson's trichrome (MT) staining were performed and analysed using a BZ-X710 all-in-one fluorescence microscope (Keyence).

Western blot analysis. Radioimmunoprecipitation assay (RIPA) buffer (50 mM Tris-HCl, $150 \mathrm{mM} \mathrm{NaCl}, 1 \mathrm{mM}$ EDTA, $0.1 \%(\mathrm{w} / \mathrm{v})$ sodium dodecyl sulfate (SDS), $0.5 \%(\mathrm{w} / \mathrm{v})$ sodium deoxycholate, $1 \%(\mathrm{v} / \mathrm{v})$ Nonidet P-40, and distilled water) with phosphatase and protease inhibitors (Roche Ltd., Basel, Switzerland) was added to the liver samples, and the mixture was homogenized using the TissueLyser LT (Qiagen, Hilden, Germany). After homogenization, the samples were centrifuged $\left(4^{\circ} \mathrm{C}, 12,000 \mathrm{x} \mathrm{g}, 15 \mathrm{~min}\right)$, and the supernatant was collected. We used western blotting to separate and quantify the proteins in homogenates of liver samples. First, protein content of the homogenates was measured using the bicinchoninic acid (BCA) method as per the manufacturer's instructions (Nacalai Tesque Inc.). Absorbance of the samples was measured at 562 nm using a microplate reader (Varioskan LUX; Thermo Fisher Scientific, Inc.). A calibration curve was prepared using bovine serum albumin (BSA) as standard (2 $\mathrm{mg} / \mathrm{ml})$; sample concentrations were determined by comparison to the standard.

Next, to separate proteins, SDS polyacrylamide gel electrophoresis (SDS-PAGE) was conducted. Each sample was diluted with sample buffer (2X Laemmli sample buffer and $10 \%$ 2-mercaptoethanol) to a concentration of $2 \mathrm{mg} / \mathrm{ml}$. Five microliters of each sample were loaded onto the gel. After electrophoresis, isolated proteins were transferred to a polyvinylidene fluoride membrane (PVDF) using Turbo blot (Bio-Rad Laboratories, Inc.). Blocking buffer containing $5 \%(\mathrm{w} / \mathrm{v})$ skim milk and 5\% (v/v) Blocking One (Nacalai Tesque Inc.) in Tris-buffered saline containing $0.1 \%$ Tween-20 (TBST) was used to block the PVDF membrane for $30 \mathrm{~min}$ at room temperature $\left(20-25^{\circ} \mathrm{C}\right)$.

Membranes were washed three times ( 5 min per wash) with TBST and allowed to incubate with the primary antibody overnight at $4^{\circ} \mathrm{C}$. Thereafter, we washed the membranes three times (5 min per wash) with TBST and incubated them with the secondary antibody (1:5,000 in TBST; Cell Signaling Technology, Inc.) for $1 \mathrm{~h}$. The PVDF membrane was washed three times and luminescence was detected using a chemiluminescent reagent (ECL Select Western Blotting Detection Reagent; GE Healthcare) and imaged using ImageQuant LAS-4000 (GE Healthcare Life Science). The signal densities were analysed using ImageJ. Table SI shows the primary and secondary antibodies used in this study.

\section{Reverse transcription-quantitative polymerase chain reaction} (RT-qPCR). The mRNA levels were measured using RT-qPCR. To extract total RNA, the liver was homogenized on ice in Sepasol-RNA I Super G (Nacalai Tesque Inc.), followed by separation into organic and aqueous phases with chloroform. Samples were allowed to sit at room temperature $\left(20-25^{\circ} \mathrm{C}\right)$ for $3 \mathrm{~min}$ and centrifuged $\left(4^{\circ} \mathrm{C}, 12,000 \mathrm{x} \mathrm{g}, 15 \mathrm{~min}\right)$. The separated aqueous phase was removed, and isopropanol was added. The mixture was mixed by inversion, incubated for $10 \mathrm{~min}$, and centrifuged $\left(4^{\circ} \mathrm{C}, 12,000 \mathrm{x} \mathrm{g}, 10 \mathrm{~min}\right)$. The supernatant was removed, and $70 \%$ ethanol was added to the pellet; the pellet was resuspended using a vortex mixer. Centrifugation 
Table I. Phenotype, blood markers, and hepatic lipid and TBARS.

\begin{tabular}{|c|c|c|c|c|c|c|c|}
\hline Measurements & WT-ND & WT-CDAHFD & KO-ND & KO-CDAHFD & Interaction & $\begin{array}{c}\text { Main } \\
\text { effect } \\
\text { (genotype) }\end{array}$ & $\begin{array}{l}\text { Main } \\
\text { effect } \\
\text { (diet) }\end{array}$ \\
\hline Final body weight (g) & $29.5 \pm 2.6$ & $23.1 \pm 1.3$ & $29.9 \pm 1.7$ & $24.0 \pm 0.9$ & n.s. & n.s. & $\mathrm{P}<0.01$ \\
\hline Liver weight/BW (mg) & $35.2 \pm 3.7$ & $61.9 \pm 10.3$ & $39.8 \pm 2.8$ & $57.2 \pm 14.6$ & n.s. & n.s. & $\mathrm{P}<0.01$ \\
\hline Serum AST activity (IU/l) & $50.3 \pm 9.6$ & $483.2 \pm 206.0$ & $54.0 \pm 7.4$ & $593.7 \pm 282.5$ & n.s. & n.s. & $\mathrm{P}<0.01$ \\
\hline Serum ALT activity (IU/l) & $33.0 \pm 7.4$ & $497.2 \pm 96.3$ & $26.8 \pm 6.8$ & $563.7 \pm 201.7$ & n.s. & n.s. & $\mathrm{P}<0.01$ \\
\hline Serum ALP activity (IU/l) & $286.8 \pm 17.5$ & $505.6 \pm 98.6$ & $237.5 \pm 23.2$ & $606.2 \pm 209.2$ & n.s. & n.s. & $\mathrm{P}<0.01$ \\
\hline Serum LDH activity (IU/1) & $300.8 \pm 54.5$ & $1,542.0 \pm 196.7$ & $535.4 \pm 59.2$ & $1,906.5 \pm 717.6$ & n.s. & n.s. & $\mathrm{P}<0.01$ \\
\hline Hepatic TCHO (mg/g) & $2.9 \pm 0.2$ & $5.4 \pm 0.5$ & $2.5 \pm 0.4$ & $5.2 \pm 0.6$ & n.s. & n.s. & $\mathrm{P}<0.01$ \\
\hline Hepatic TG (mg/g) & $18.5 \pm 3.9$ & $300.9 \pm 27.8$ & $13.9 \pm 4.6$ & $304.2 \pm 33.6$ & n.s. & n.s. & $\mathrm{P}<0.01$ \\
\hline Hepatic TBARS (nmol/mg) & $1.4 \pm 0.5$ & $7.3 \pm 3.3$ & $1.8 \pm 0.2$ & $7.9 \pm 2.2$ & n.s. & $\mathrm{P}=0.06$ & $\mathrm{P}<0.01$ \\
\hline
\end{tabular}

Data are shown as presented as the mean $\pm \mathrm{SD}$. AST, aspartate aminotransferase; ALT, alanine aminotransferase; ALP, alkaline phosphatase; LDH, lactate dehydrogenase; TCHO, total cholesterol; TG, triglycerides; TBARS, thiobarbituric acid reactive substances; CDAHFD, choline-deficient high-fat diet; WT, wild type; KO, renalase knock out; ND, normal diet.

$\left(4^{\circ} \mathrm{C}, 12,000 \mathrm{x} \mathrm{g}, 5 \mathrm{~min}\right)$ was performed to remove the supernatant. Finally, UltraPure DNase/RNase-Free Distilled Water (Invitrogen; Thermo Fisher Scientific, Inc.) was added, and the sample was incubated at $65^{\circ} \mathrm{C}$ for $5 \mathrm{~min}$. The extracted RNA was quantified using spectrophotometry (Nanodrop ND2000, Thermo Fisher Scientific, Inc.). The extracted RNA was used for cDNA synthesis using PrimeScript RT reagent kit (Takara Bio) was performed as per the manufacturer's instructions. Reverse transcription was carried out in a thermal cycler (TP 350; Takara Bio) $\left(37^{\circ} \mathrm{C}, 15 \mathrm{~min} ; 85^{\circ} \mathrm{C}, 5 \mathrm{sec} ; 4^{\circ} \mathrm{C}, \infty\right)$. After reverse transcription, the cDNA was diluted 10 -fold using UltraPure DNase/RNase-Free Distilled Water. KAPA SYBR FAST qPCR Master Mix (KK 4602; Kapa Biosystems), upstream and downstream primers, ROX Low Reference Dye (KD 4601; Kapa Biosystems), and UltraPure Distilled Water were added, and PCR amplification was conducted using a QuantStudio 5 Real-Time PCR System (Thermo Fisher Scientific). RT-qPCR amplification was set up as follows: initial denaturation at $95^{\circ} \mathrm{C}$ for $20 \mathrm{sec}$, followed by 35 cycles of denaturation at $95^{\circ} \mathrm{C}$ for $30 \mathrm{sec}$ and annealing and extension at $60{ }^{\circ} \mathrm{C}$ for $30 \mathrm{sec}$. The mRNA level of the TATA-binding protein (encoded by the mouse $T b p$ gene) was used as the housekeeping gene. The cycle threshold $(\mathrm{Ct})$ value of the target gene was standardized to that of the housekeeping gene $(\Delta \Delta \mathrm{Ct}$ method) (19). The relative expression level of the target gene was calculated as the relative value compared with that of the WT-ND group. The primer sequences used in this experiment are shown in Table SII.

Thiobarbituric acid reactive substances (TBARS). TBARS measurement in the liver was performed as previously described (20). Briefly, PBS with a protease inhibitor (Roche Ltd.) was added to the tissue. The tissue was crushed with a bead crusher and centrifuged, and the supernatant was used as the sample. SDS (5.2\%), thiobarbituric acid solution (0.8\%), ultrapure water, butylhydroxyltriene glacial acetic acid solution $(0.8 \%)$ were mixed in a ratio of $4: 30: 34: 1$ to prepare the reaction solution. Ethanol $(10 \%, 997.8 \mu \mathrm{l})$ was added to $2.2 \mu \mathrm{l}$ of 1,1,3,3-tetraethoxypropane (TEP), and this solution was diluted 100 times with ultrapure water to prepare a $100 \mathrm{nmol} / \mathrm{ml}$ TEP solution (standard solution). Furthermore, this solution was diluted with ultrapure water to prepare standard solutions for a calibration curve ranging from 0 to $50 \mathrm{nmol} / \mathrm{ml}$.

The reaction solution and $0.1 \mathrm{M}$ acetate buffer were poured into a $1.5-\mathrm{ml}$ tube, and the sample or standard solution was added. After allowing to sit still at $4^{\circ} \mathrm{C}$ for $30 \mathrm{~min}$, the solution was subjected to heat shock at $100^{\circ} \mathrm{C}$ for $60 \mathrm{~min}$. Butanol:pyridine (15:1, v/v) was added to the solutions on ice. The mixture was vigorously stirred and centrifuged $(300 \mathrm{x} \mathrm{g}$, $10 \mathrm{~min}, 4^{\circ} \mathrm{C}$ ). The supernatant was transferred into a 96 -well microplate, and the absorbance at $532 \mathrm{~nm}$ was measured using a microplate reader (Varioskan LUX; Thermo Fisher Scientific, Inc.).

A calibration curve was created based on the absorbance of the standard solutions, and the TBARS concentration in the sample was determined. The TBARS concentration was expressed per mg of protein.

Statistical analysis. Data are presented as mean \pm SD. For almost measurements, a two-way analysis of variance was conducted. For body weight data, a three-way ANOVA was conducted. In the case of significant interaction between the genotype and diet (and time), comparisons were made using Bonferroni's post hoc test. GraphPad Prism 8 software (GraphPad Software, Inc.) was used for all statistical calculations, and the significance level was set to $\mathrm{P}<0.05$ or $\mathrm{P}<0.01$ for all cases.

\section{Results}

Effects of the renalase $K O$ and CDAHFD on overall mouse health and the liver status. After feeding ND or CDAHFD for 6 weeks starting from 5 weeks of age, WT and KO mice given ND exhibited significant increase in body weight from 6 or 7 weeks of age. However, the CDAHFD group did not show any significant change in body weight. Nonetheless, there was a significant difference between the final body weights 


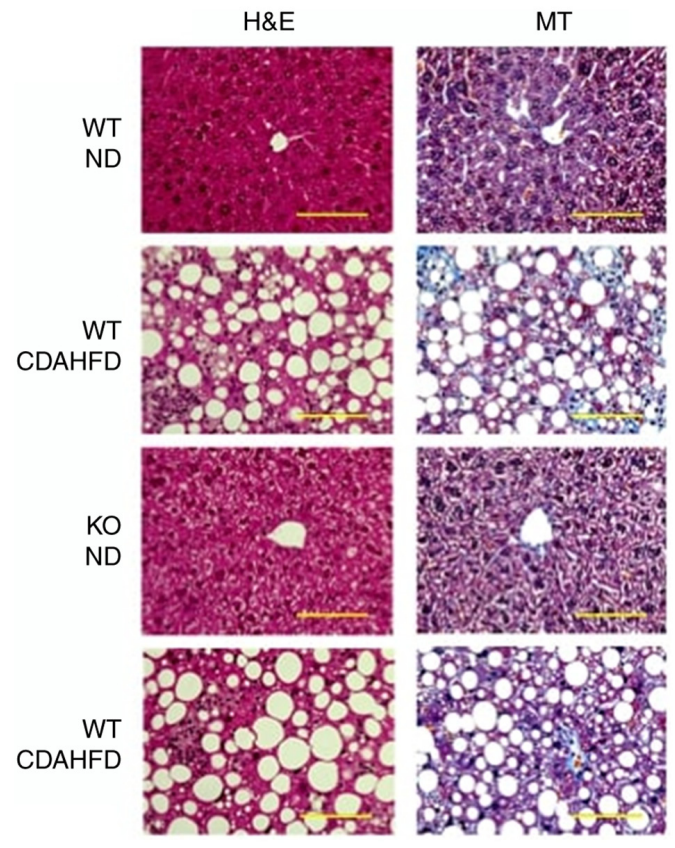

Figure 1. Evaluation of hepatic histology on NASH model mice. The H\&E (Left) and MT (Right) stained histology images of the liver in each group are shown. Scale bar $=100 \mu \mathrm{m}$. WT, wild type; KO, renalase knock out; MS, Masson's trichrome; H\&E, hematoxylin eosin; CDAHFD, choline-deficient high-fat diet; NASH, nonalcoholic steatohepatitis.
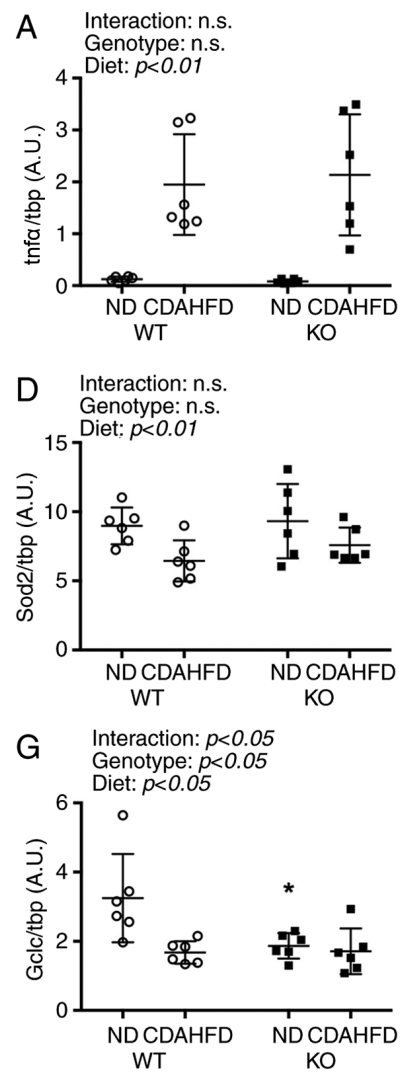
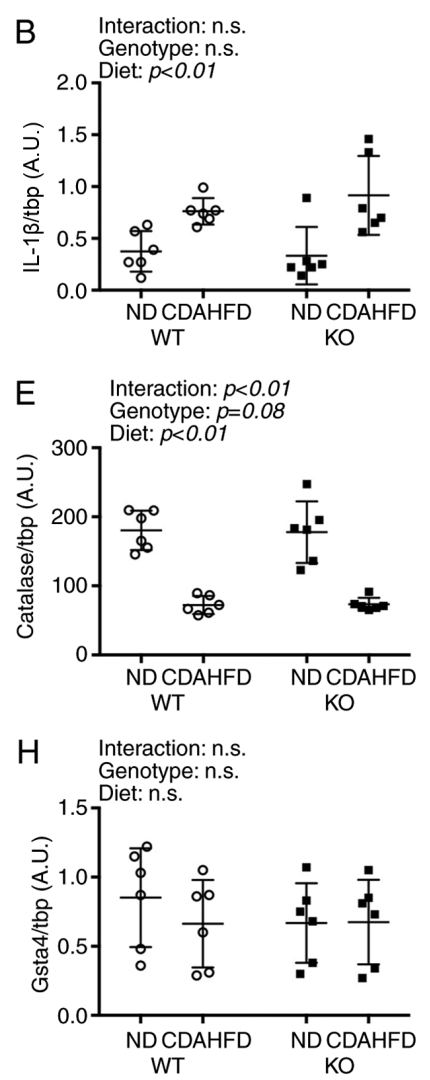
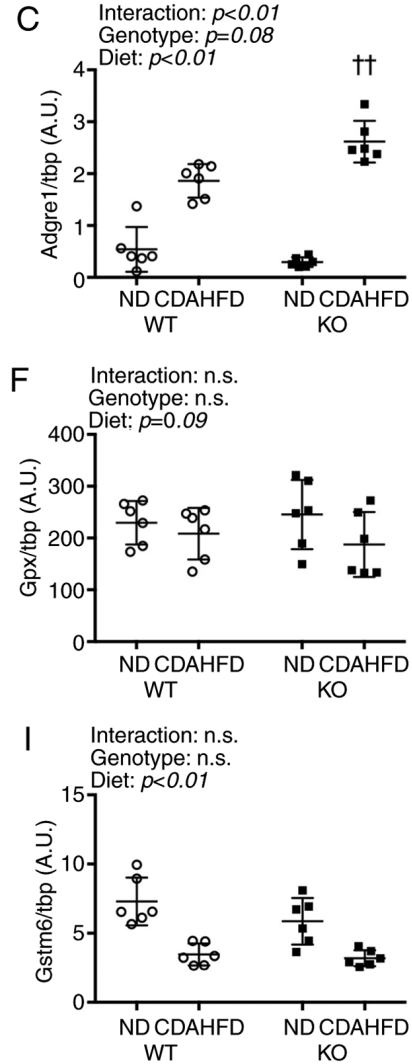

Figure 2. Inflammation and antioxidant mRNA expressions in liver on NASH model mice. (A-I) All mRNA expressions were compared between WT and KO fed ND or CDAHFD groups using the $\Delta \Delta \mathrm{Cq}$ method. Data are shown as mean $\pm \mathrm{SD}$. $\mathrm{n}=6$ in each group. Data were analyzed using two way ANOVA. $\mathrm{P}<0.05$ vs. WT-ND. ${ }^{\dagger} \mathrm{P}<0.01$ vs. WT-CDAHFD. WT, wild type; KO, renalase knock out; tnf $\alpha$, tumor necrosis factor- $\alpha$; IL-1 $\beta$, interleukin-1 $\beta$; Adgre1, adhesion G protein-coupled receptor E1; Sod, superoxide disumtase; Gpx, gluthatione peroxidase; Gclc, $\gamma$-glutamylcysteine synthetase; Gsta4, gluthatione S-transferase $\alpha 4$; Gstm6, gluthatione S-transferase mu 6; CDAHFD, choline-deficient high-fat diet; NASH, nonalcoholic steatohepatitis; ND, normal diet.

of mice in the ND and CDAHFD groups (Table I). We also show the trend in body weight change throughout the study
(Fig. S1A). In addition, renalase mRNA levels in the liver were measured in all mice (Fig. S1B). 

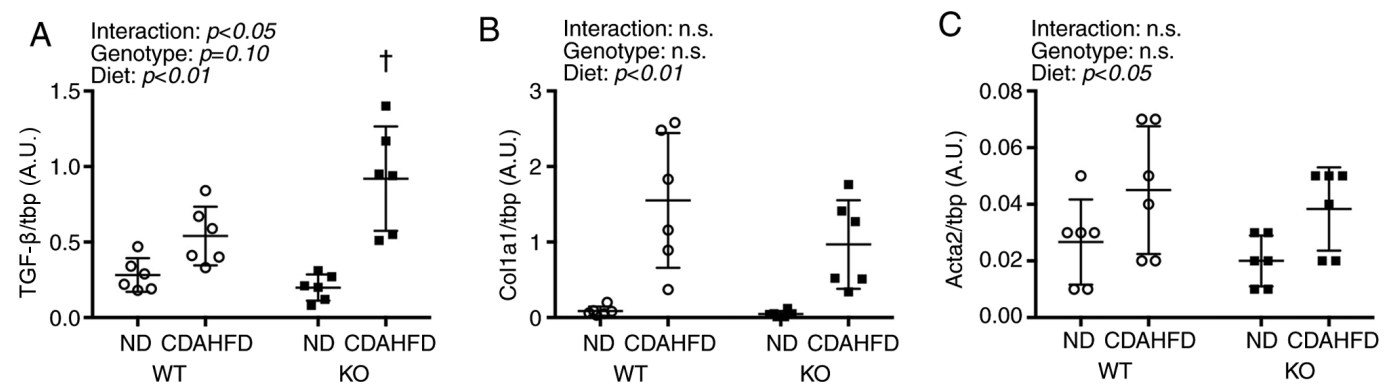

Figure 3. Fibrosis mRNA expressions in liver on NASH model mice. mRNA expression of (A) Tgfb1, (B) Colla1 and (C) Acta2 were compared between WT and KO fed ND or CDAHFD groups using the $\Delta \Delta \mathrm{Ct}$ method. Data are shown as mean $\pm \mathrm{SD}$. Data were analyzed using two way ANOVA. ${ }^{\mathrm{P}}<0.05$ vs. WT-CDAHFD. WT, wild type; KO, renalase knock out; TGF- $\beta$, transforming growth factor- $\beta$; Colla1, collagen type I $\alpha 1$ chain; Acta2, actin $\alpha 2$; CDAHFD, choline-deficient high-fat diet; NASH, nonalcoholic steatohepatitis; ND, normal diet.
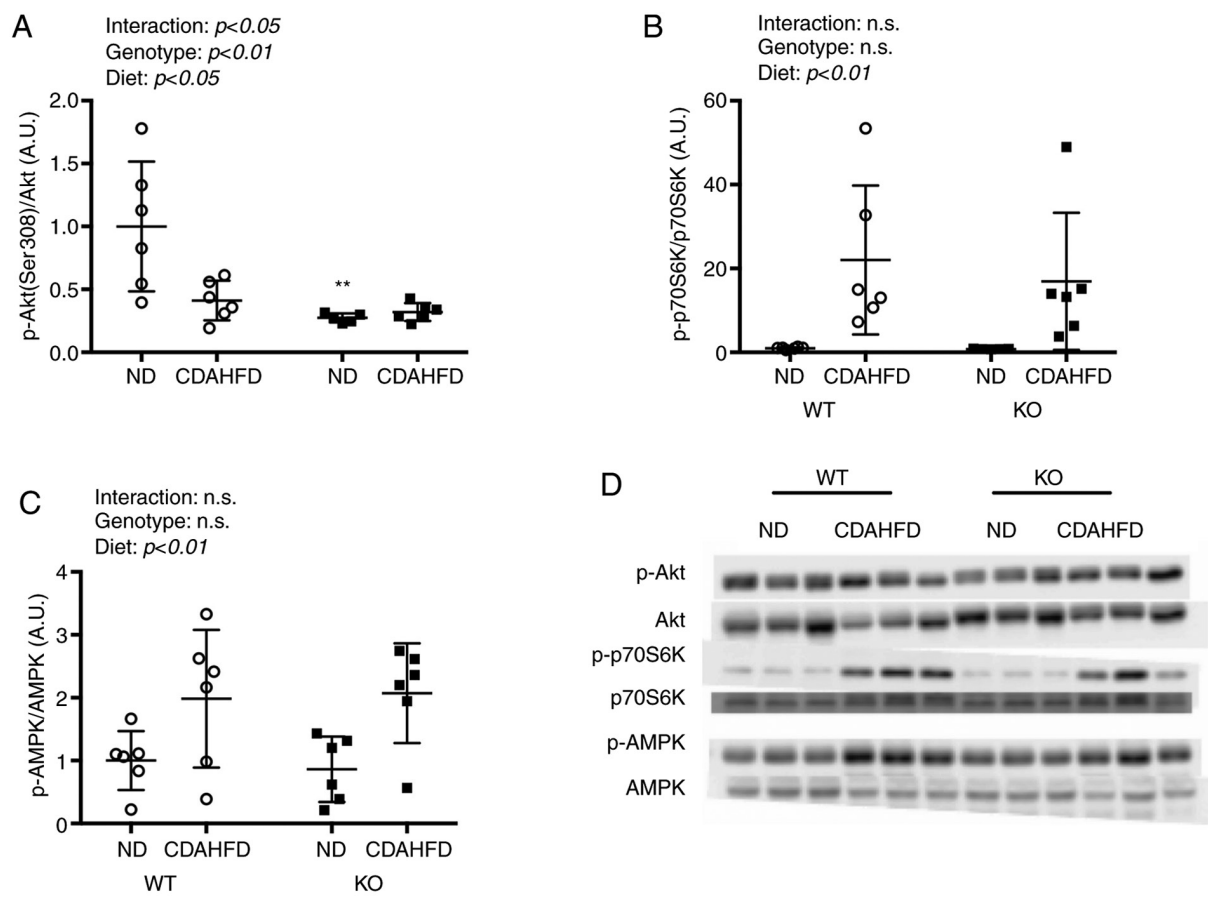

Figure 4. Akt, p70S6K, AMPK expressions in liver on NASH model mice. Protein expressions of (A) Ser308, (B) p70S6K and (C) AMPK were compared between WT and KO fed ND or CDAHFD groups using the western blotting method. (D) Western blot analysis. Data are shown as mean \pm SD. Data were analyzed using two way ANOVA. ${ }^{* *} \mathrm{P}<0.01$ vs. WT-ND. WT, wild type; KO, renalase knock out; p, phosphorylated; CDAHFD, choline-deficient high-fat diet; NASH, nonalcoholic steatohepatitis; ND, normal diet; p70S6K, p70S6 kinase.

Furthermore, CDAHFD significantly increased liver weight compared with ND (Table I). Activities of serum AST, ALT, and ALP, which are liver function markers in the blood, also increased following CDAHFD. CDAHFD resulted in a significantly higher serum LDH than that by ND in both WT and $\mathrm{KO}$ mice. Significant elevations in T-CHO and TG in the liver were observed in mice under CDAHFD. Similarly, concentration of TBARS and lipid peroxide increased as an effect of CDAHFD.

Hepatic lipid accumulation and fibrosis. Based on H\&E staining, significant accumulation of lipid droplets was observed in mice under CDAHFD, but no significant difference was observed between the histology of mice in the WT-CDAHFD and KO-CDAHFD groups (Fig. 1).

MT staining showed collagen fibres stained in blue in the liver of mice under CDAHFD, but no significant difference was observed between the histology of mice in the WT-CDAHFD and KO-CDAHFD groups (Fig. 1).

$m R N A$ levels of inflammatory and oxidative stress markers in the liver. Increased transcript levels of inflammatory cytokines Tnf $\alpha$ and IL-1 $\beta$ were observed under CDAHFD (Fig. 2). Furthermore, the mRNA level of the adhesion G protein-coupled receptor E1 (Adgre1), a macrophage marker, was found to be significantly higher in the KO-CDAHFD group than in the WT-CDAHFD group.

The mRNA levels of antioxidative enzymes superoxide dismutase 2 ( $\operatorname{Sod} 2)$, which is found in the mitochondria, and catalase decreased following CDAHFD. Moreover, glutathione peroxidase (Gpx) mRNA levels were not affected by the type of diet. The mRNA level of the $\gamma$-glutamylcysteine synthetase catalytic unit (Gclc), which is involved in the synthesis of glutathione, an antioxidant, was affected by the genotype 

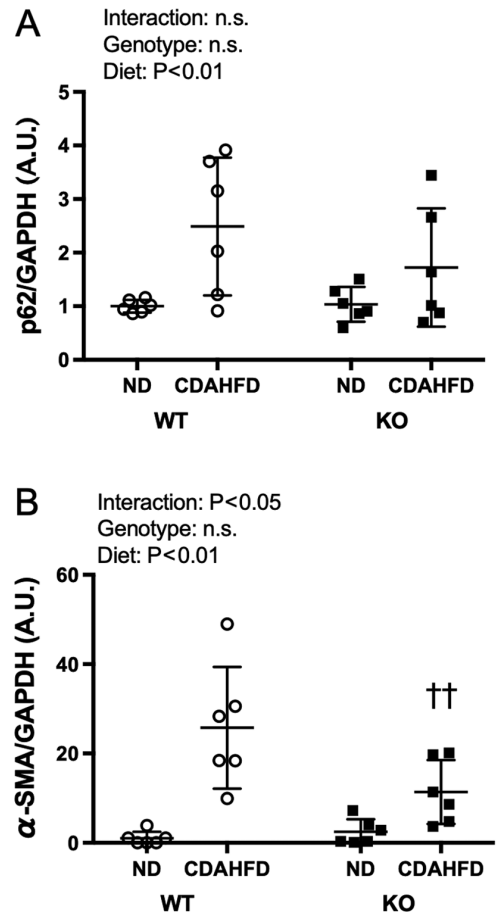

\section{C}
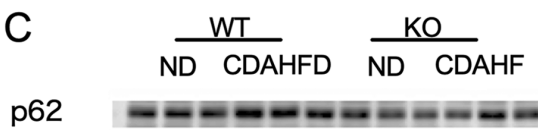

$\alpha$-SMA

GAPDH

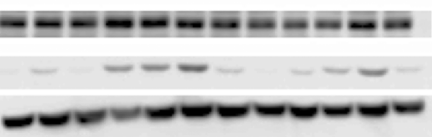

Figure 5. $\alpha$-SMA and p62 protein expressions in liver on NASH model mice. Protein expressions of (A p62 and (B) $\alpha$-SMA were compared between WT and KO fed ND or CDAHFD groups using western blotting. (C) Image of western blot bands. Data are shown as mean \pm SD. Data were analyzed using two way ANOVA. ${ }^{\circ} \mathrm{P}<0.01$ vs. WT-CDAHFD. WT, wild type; KO, renalase knock out; NASH, nonalcoholic steatohepatitis; CDAHFD, choline-deficient high-fat diet; ND, normal diet; $\alpha$-SMA, $\alpha$-smooth muscle actin.

and diet and was significantly lower in the KO-ND group than in the WT-ND group. Furthermore, CDAHFD significantly reduced the transcript levels of glutathione S-transferase mu 6 (Gstm6) but did not exert significant effects on that of glutathione S-transferase $\alpha 4$ (Gsta4).

mRNA levels of liver fibrosis markers. An interaction was observed between $T g f b l$ and diet, and the KO-CDAHFD group had significantly higher $T g f b l$ mRNA levels than the WT-CDAHFD group (Fig. 3). Furthermore, CDAHFD increased the levels of Collal and Acta2 mRNA.

Protein expression of $A M P K, A k t$, and p70S6K. There was a significant interaction between the diet and genotype for the levels of phosphorylated Akt (p-Akt) (Fig. 4). The p-Akt/Akt levels in the WT-ND group were significantly higher than that in the KO-ND group. In addition, CDAHFD led to a significant increase in the phosphorylation of p70S6K (p-p70S6K). Phosphorylation of AMPK also increased with CDAHFD.

Expression levels of p62 and $\alpha$-SMA. For the expression level of $\alpha$-SMA, an interaction between the diet and genotype was observed; $\alpha$-SMA expression in the KO-CDAHFD group was significantly lower than that in the WT-CDAHFD group (Fig. 5).

\section{Discussion}

A methionine/choline deficient diet (MCD) is one of the ways to induce NASH in rodents $(7,9)$. However, the MCD mouse model experiences body weight loss accompanied by weight loss of white adipose tissue, which increases the risk of mortality (9). Therefore, a novel diet-induced NASH model using CDAHFD has been established as it exhibits fat accumulation and fibrosis in the liver and suppresses the weight loss observed in the MCD model (9). In the present study, the CDAHFD model was selected as the NASH model. Our findings showed that there was no difference in the body weight between WT and KO mice, although the body weight of the mice in the CDAHFD groups was significantly lower than that of the mice in the ND groups. Furthermore, the levels of liver function markers in the blood (serum AST, ALT, ALP, and LDH activities), as well as T-CHO and TG levels significantly increased as an effect of the CDAHFD. Moreover, accumulation of lipid droplets and collagen fibres were confirmed by $\mathrm{H} \& \mathrm{E}$ and MT staining, respectively, in the WT-CDAHFD and KO-CDAHFD groups. These observations are consistent with those in previous studies $(6,8)$. Suga et al observed that mice fed with CDAHFD for 6 weeks show hepatic steatosis and fibrosis (8). However, in the present study, significant effects of the renalase $\mathrm{KO}$ were not observed on the body weight, liver weight, blood liver function markers, and liver lipid levels. Furthermore, there was no difference between the H\&E and MT staining for the WT-CDAHFD and KO-CDAHFD groups. Therefore, renalase deficiency did not have dramatic effects on the NASH pathology in this study.

In NAFLD and NASH, oxidative stress is enhanced by mitochondrial abnormalities, endoplasmic reticulum stress, and endotoxin action; liver fibrosis progresses through the activation of hepatic stellate cells (5-9). Renalase has been reported to inhibit oxidative stress (21). Therefore, in order to evaluate the effect of renalase deficiency on oxidative stress in the NASH model, the levels of TBARS and mRNA levels of genes involved in glutathione metabolism and regula- 
tion of oxidative stress were measured. Moreover, hepatic TBARS concentration is used for evaluating the amount of lipid peroxides and as an index of oxidative stress $(20,22)$. We found hepatic TBARS concentration to be significantly higher in the CDAHFD groups than in the ND groups. This is consistent with an increase in the liver reactive oxygen species and TBARS concentrations in other studies on NASH models $(6,7,23,24)$. In contrast, we could partly observe the expected increase the levels of TBARS in the mice with renalase KO. The hepatic TBARS in KO mice might be higher than that in WT mice because of the mouse genotypes considered in this study. Additionally, the Gclc mRNA level was significantly lower in the KO-ND group than in the WT-ND group. $\gamma$-glutamylcysteine ligase, the rate-limiting enzyme for glutathione synthesis, contributes to the maintenance of the intracellular redox balance by glutathione synthesis (25). Therefore, based on the low levels of Gclc transcripts in the $\mathrm{KO}$ mice, we infer that the $\mathrm{KO}$ mice had low intracellular GSH levels due to the inhibition of glutathione synthesis. Moreover, when CDAHFD is ingested for a period longer than 6 weeks as in this experiment, the renalase KO may increase oxidative stress and affect NASH pathology.

Macrophages present in the liver are divided into Kupffer cells and monocyte-derived macrophages, and monocyte-derived macrophages infiltrate the liver tissues in response to chemokines secreted from hepatocytes and Kupffer cells (26). In our study, transcription of the Adgre1 gene, which encodes F4/80, a marker of mature macrophages, was significantly higher in the KO-CDAHFD group than in the WT-CDAHFD group. In other words, hepatocyte dysfunction might have occurred in the absence of renalase. The secretion of inflammatory cytokines and chemokines in tissues may have been promoted through the activation of the $\mathrm{NF}-\kappa \mathrm{B}$ pathway, and the migration of macrophages into the liver may be due to oxidative stress suppression and mitochondrial mitotic inhibitory effect caused by the renalase deficiency $(22,27,28)$. Alternatively, it is possible that the expression of Kupffer cells will have increased in the renalase $\mathrm{KO}$ mice; however, this needs further investigation. Moreover, NF- $\kappa \mathrm{B}$-regulated renalase expression led to phosphorylation of Akt via PMCA $4 \mathrm{~b}$ receptor, although no effect of hepatic fibrosis induced by oxidative stress on inflammation via $N F-\kappa B$ was observed in the MT staining of WT and KO mice. Furthermore, it has been reported that phosphorylation of Akt is caused by hepatic stellate cell (29). We observed that phosphorylation of Akt in WT mice decreased significantly compared with that in $\mathrm{KO}$ mice. Therefore, the decreased expression of fibrosis marker $\alpha$-SMA in KO mice might result from the decreased p-Akt in this study.

The expression level of the $T g f b l$ gene was significantly higher in the KO-CDAHFD group than in the WT-CDAHFD group. Furthermore, the expression levels of type I collagen fibres and $\alpha$-SMA are expected to increase through the TGF- $\beta /$ Smad pathway mediated by the TGF- $\beta$ receptor (30). However, the expression levels of $\alpha$-SMA were lower in the KO-CDAHFD group than in the WT-CDAHFD group, while the Acta2 mRNA levels were not significantly different between the WT-CDAHFD and the KO-CDAHFD groups. This may be because of the suppression of the signal down- stream of the TGF- $\beta$ receptor. Therefore, further investigation is needed to evaluate the effects of it on the activation of SMAD2/3, which regulates the production of extracellular matrix proteins through the TGF- $\beta$ signalling pathway and SMURF2 (31), which is known to suppress the SMAD2/3 signalling pathway.

In conclusion, renalase deficiency had no significant effects on NASH pathology in the CDAHFD-administered NASH mouse model. However, our findings suggest that hepatic fibrosis progression may be partly suppressed, while oxidative stress increases and macrophage infiltration occurs in the absence of renalase. Therefore, our study suggests renalase in the liver, as a potential therapeutic target for fibrosis in NASH (Fig. 6). In the future study, it will be also needed to measure fibronectin 1 and colla1 protein expression because these proteins are fibrosis markers (32).

\section{Acknowledgements}

Not applicable.

\section{Funding}

No funding was received.

\section{Availability of data and materials}

All data generated or analysed during this study are included in this published article.

\section{Authors' contributions}

KTo, TK and KTa designed the concept of the current study. KTo, NS, KA and SO performed the experiments. KTo drafted the manuscript. KTo, NS, KA, TK, YY, TS and KTa analyzed the data. All authors read and revised the manuscript. All authors read and approved the final manuscript.

\section{Ethics approval and consent to participate}

All experiments in the present study were approved by the Animal Subjects Committee, University of Tsukuba, Japan (approval no. 19-455).

\section{Patient consent for publication}

Not applicable.

\section{Competing interests}

The authors declare that they have no competing interests.

\section{References}

1. Bugianesi E, Leone N, Vanni E, Marchesini G, Brunello F, Carucci P, Musso A, De Paolis P, Capussotti L, Salizzoni M, et al: Expanding the natural history of nonalcoholic steatohepatitis: From cryptogenic cirrhosis to hepatocellular carcinoma. Gastroenterology 123: 134-140, 2002.

2. Esler WP and Bence KK: Metabolic Targets in Nonalcoholic Fatty Liver Disease. Cell Mol Gastroenterol Hepatol 8: 247-267, 2019. 
3. Tilg $\mathrm{H}$ and Moschen AR: Evolution of inflammation in nonalcoholic fatty liver disease: The multiple parallel hits hypothesis. Hepatology 52: 1836-1846, 2010.

4. Day CP and James OFW: Steatohepatitis: A tale of two 'hits'? Gastroenterology 114: 842-845, 1998 .

5. Anty R and Gual P: Pathogenesis of non-alcoholic fatty liver disease. Presse Med 48: 1468-1483, 2019 (In French).

6. Takatani N, Kono Y, Beppu F, Okamatsu-Ogura Y, Yamano Y, Miyashita K and Hosokawa M: Fucoxanthin inhibits hepatic oxidative stress, inflammation, and fibrosis in diet-induced nonalcoholic steatohepatitis model mice. Biochem Biophys Res Commun 528: 305-310, 2020.

7. Ke Z, Zhao Y, Tan S, Chen H, Li Y, Zhou Z and Huang C: Citrus reticulata Blanco peel extract ameliorates hepatic steatosis, oxidative stress and inflammation in HF and MCD diet-induced NASH C57BL/6 J mice. J Nutr Biochem 83: 108426, 2020.

8. Suga T, Yamaguchi H, Ogura J, Shoji S, Maekawa M and Mano N: Altered bile acid composition and disposition in a mouse model of non-alcoholic steatohepatitis. Toxicol Appl Pharmacol 379: $114664,2019$.

9. Matsumoto M, Hada N, Sakamaki Y, Uno A, Shiga T, Tanaka C, Ito T, Katsume A and Sudoh M: An improved mouse model that rapidly develops fibrosis in non-alcoholic steatohepatitis. Int J Exp Pathol 94: 93-103, 2013.

10. Xu J, Li G, Wang P, Velazquez H, Yao X, Li Y, Wu Y, Peixoto A, Crowley $\mathrm{S}$ and Desir GV: Renalase is a novel, soluble monoamine oxidase that regulates cardiac function and blood pressure. J Clin Invest 115: 1275-1280, 2005

11. Wu Y, Xu J, Velazquez H, Wang P, Li G, Liu D, Sampaio-Maia B, Quelhas-Santos J, Russell K, Russell R, et al: Renalase deficiency aggravates ischemic myocardial damage. Kidney Int 79: 853-860, 2011

12. Wang F, Huang B, Li J, Liu L and Wang N: Renalase might be associated with hypertension and insulin resistance in type 2 diabetes. Ren Fail 36: 552-556, 2014.

13. Wang L, Velazquez H, Chang J, Safirstein R and Desir GV: Identification of a receptor for extracellular renalase. PLoS One 10: e0122932, 2015.

14. Wang Y, Safirstein R, Velazquez H, Guo XJ, Hollander L, Chang J, Chen TM, Mu JJ and Desir GV: Extracellular renalase protects cells and organs by outside-in signalling. J Cell Mol Med 21: 1260-1265, 2017

15. Wu Y, Wang L, Deng D, Zhang Q and Liu W: Renalase protects against renal fibrosis by inhibiting the activation of the ERK signaling pathways. Int J Mol Sci 18: 1-25, 2017

16. Li H, Guo J, Liu H, Niu Y, Wang L, Huang K and Wang J: Renalase as a novel biomarker for evaluating the severity of hepatic ischemia-reperfusion injury. Oxid Med Cell Longev 2016 $3178562,2016$.

17. Zhang T, Gu J, Guo J, Chen K, Li H and Wang J: Renalase attenuates mouse fatty liver ischemia/reperfusion injury through mitigating oxidative stress and mitochondrial damage via activating SIRT1. Oxid Med Cell Longev 2019: 7534285, 2019.

18. Nagate T, Chino T, Nishiyama C, Okuhara D, Tahara T, Maruyama Y, Kasahara H, Takashima K, Kobayashi S, Motokawa Y, et al: Diluted isoflurane as a suitable alternative for diethyl ether for rat anaesthesia in regular toxicology studies. J Vet Med Sci 69: 1137-1143, 2007.
19. Livak KJ, Schmittgen TD. Analysis of relative gene expression data using real-time quantitative PCR and the $2-\Delta \Delta C T$ method. Methods. 2001:25(4):402-408. doi:10.1006/meth.2001.1262

20. Kikugawa K, Yasuhara Y, Ando K, Koyama K, Hiramoto K and Suzuki M: Effect of supplementation of $n-3$ polyunsaturated fatty acids on oxidative stress-induced DNA damage of rat hepatocytes. Biol Pharm Bull 26: 1239-1244, 2003.

21. Zhao B, Zhao Q, Li J, Xing T, Wang F and Wang N: Renalase protects against contrast-induced nephropathy in Sprague-Dawley rats. PLoS One 10: e0116583, 2015.

22. Ghani MA, Barril C, Bedgood DR Jr and Prenzler PD Measurement of antioxidant activity with the thiobarbituric acid reactive substances assay. Food Chem 230: 195-207, 2017.

23. Palladini G, Di Pasqua LG, Berardo C, Siciliano V, Richelmi P, Perlini S, Ferrigno A and Vairetti M: Animal models of steatosis (NAFLD) and steatohepatitis (NASH) exhibit hepatic lobe-specific gelatinases activity and oxidative stress. Can J Gastroenterol Hepatol 2019: 5413461, 2019.

24. Horas H Nababan S, Nishiumi S, Kawano Y, Kobayashi T, Yoshida M, Azuma T and Azuma T: Adrenic acid as an inflammation enhancer in non-alcoholic fatty liver disease. Arch Biochem Biophys 623-624: 64-75, 2017.

25. Chen Y, Dong H, Thompson DC, Shertzer HG, Nebert DW and Vasiliou V: Glutathione defense mechanism in liver injury: Insights from animal models. Food Chem Toxicol 60: 38-44, 2013.

26. Cha JY, Kim DH and Chun KH: The role of hepatic macrophages in nonalcoholic fatty liver disease and nonalcoholic steatohepatitis. Lab Anim Res 34: 133-139, 2018.

27. Wu Y, Wang L, Wang X, Wang Y, Zhang Q and Liu W: Renalase contributes to protection against renal fibrosis via inhibiting oxidative stress in rats. Int Urol Nephrol 50: 1347-1354, 2018.

28. Huang Z, Li Q, Yuan Y, Zhang C, Wu L, Liu X, Cao W, Guo H, Duan S, Xu X, et al: Renalase attenuates mitochondrial fission in cisplatin-induced acute kidney injury via modulating sirtuin-3. Life Sci 222: 78-87, 2019.

29. Cai CX, Buddha H, Castelino-Prabhu S, Zhang Z, Britton RS, Bacon BR and Neuschwander-Tetri BA: Activation of insulin-PI3K/Akt-p70S6K pathway in hepatic stellate cells contributes to fibrosis in nonalcoholic steatohepatitis. Dig Dis Sci 62: 968-978, 2017.

30. Xu F, Liu C, Zhou D and Zhang L: TGF- $\beta /$ SMAD Pathway and Its Regulation in Hepatic Fibrosis. J Histochem Cytochem 64: 157-167, 2016.

31. Ma L, Li H, Zhang S, Xiong X, Chen K, Jiang P, Jiang K and Deng G: Emodin ameliorates renal fibrosis in rats via TGF- $31 /$ Smad signaling pathway and function study of Smurf 2. Int Urol Nephrol 50: 373-382, 2018.

32. Lv Y, Bing Q, Lv Z, Xue J, Li S, Han B, Yang Q, Wang X and Zhang Z: Imidacloprid-induced liver fibrosis in quails via activation of the TGF- $\beta 1 /$ Smad pathway. Sci Total Environ 705: 135915,2020

This work is licensed under a Creative Commons Attribution-NonCommercial-NoDerivatives 4.0 International (CC BY-NC-ND 4.0) License. 